\title{
Genetic polymorphisms of organic cation transporters 1 (OCT1) and responses to metformin therapy in individuals with type 2 diabetes mellitus: a systematic review protocol
}

Edith Pascale Mofo Mato ${ }^{1 *}$ (D, Magellan Guewo-Fokeng ${ }^{2,3}$, M. Faadiel Essop ${ }^{4}$ and Peter Mark Oroma Owira ${ }^{1}$

\begin{abstract}
Background: Metformin is one of the most commonly used drugs for type 2 diabetes mellitus (T2DM). Despite its efficacy and safety, metformin is frequently associated with highly variable glycemic responses, which is hypothesized to be the result of genetic variations in its transport by organic cation transporters (OCTs). This systematic review aims to highlight and summarize the overall effects of OCT1 polymorphisms on therapeutic responses to metformin and to evaluate their potential role in terms of interethnic differences with metformin responses.

Methods/design: We will systematically review observational studies reporting on the genetic association between OCT1 polymorphisms and metformin responses in T2DM patients. A comprehensive search strategy formulated with the help of a librarian will be used to search MEDLINE via PubMed, Embase, and CINAHL for relevant studies published between January 1990 and July 2017. Two review authors will independently screen titles and abstracts in duplicate, extract data, and assess the risk of bias with discrepancies resolved by discussion or arbitration of a third review author. Mined data will be grouped according to OCT1 polymorphisms, and their effects on therapeutic responses to metformin will be narratively synthesized. If sufficient numbers of homogeneous studies are scored, meta-analyses will be performed to obtain pooled effect estimates. Funnel plots analysis and Egger's test will be used to assess publication bias. This study will be reported according to the Preferred Reporting Items for Systematic Review and Meta-Analysis (PRISMA) guidelines. Discussion: This review will summarize the genetic effects of OCT1 polymorphisms associated with variabilities in glycemic responses to metformin. The findings of this study could help to develop genetic tests that could predict a person's response to metformin treatment and create personalized drugs with greater efficacy and safety.
\end{abstract}

Systematic review registration: Registration number: PROSPERO, CRD42017079978

Keywords: Genetic polymorphisms, Glycemic response, Metformin, OCT1, Type 2 diabetes mellitus

\footnotetext{
* Correspondence: edithmofo8818@yahoo.fr

${ }^{1}$ Molecular and Clinical Pharmacology Research Laboratory, Department of

Pharmacology, Discipline of Pharmaceutical Sciences, School of Health

Sciences, University of KwaZulu-Natal, PO Box X5401, Durban, South Africa

Full list of author information is available at the end of the article
}

(c) The Author(s). 2018 Open Access This article is distributed under the terms of the Creative Commons Attribution 4.0 International License (http://creativecommons.org/licenses/by/4.0/), which permits unrestricted use, distribution, and reproduction in any medium, provided you give appropriate credit to the original author(s) and the source, provide a link to the Creative Commons license, and indicate if changes were made. The Creative Commons Public Domain Dedication waiver (http://creativecommons.org/publicdomain/zero/1.0/) applies to the data made available in this article, unless otherwise stated. 


\section{Background}

Metformin is recommended by major clinical practice guidelines as first-line oral anti-hyperglycemic drug for use as monotherapy in individuals with newly diagnosed T2DM [1]. It specifically decreases hepatic gluconeogenesis without enhancing insulin secretion, inducing weight gain, or increasing the risk of hypoglycemia [2].

The precise molecular mechanisms underlying metformin's action are not well understood. It was initially suggested that one of the key actions of metformin is to activate AMP-activated protein kinase (AMPK) through a decrease in hepatic energetic status (increasing AMP: $\mathrm{ADP}$ and/or ADP/ATP concentration ratios) or through an upstream AMPK kinase (LKB1), leading to lowered transcription of gluconeogenic genes [3]. However, recent investigations in conditional AMPK knockout mice showed that metformin inhibits hepatic gluconeogenesis in an LKB1- and AMPK-independent manner via a decrease in hepatic energetic status $[4,5]$. This preferential action of metformin in hepatocytes is due to the predominant expression of organic cation transporter 1 (OCT1), the main transporter responsible for hepatic uptake of metformin [6, 7].

OCT1 belongs to the solute carriers (SLC) 22A superfamily of polyspecific membrane proteins that play a central role in transportation of organic cations, anions, and zwitterions thus playing a major role in the cellular organic ions homeostasis [8, 9]. In human, OCT1 is most strongly expressed in the liver, whereas in rodents, OCT1 is also strongly expressed in the kidney and small intestine. In human and rat liver, OCT1 is located to the sinusoidal membrane of the hepatocytes [10]. The major function of OCT1 most likely is mediating the uptake of organic cations in hepatocytes as the initial step of biliary secretion [11].

Despite its widespread use, there are considerable variations in response to metformin therapy ranging from improvement in HbA1c levels (by up to 4\%) to estimates of $\sim 35 \%$ failure to achieve the glycemic goal (HbA1c level less than 7\%) [12]. These variabilities in metformin efficacy clearly suggest the implication of individual genetic imprints [13]. Since genetic variations in some drug transporters can dramatically alter the pharmacokinetics and pharmacodynamics of many drugs, studies conducted in different population groups have suggested that OCT1 genetic polymorphisms could affect metformin responses $[14,15]$. However, there is no consensus about its precise effect; both positive and negative findings have been reported [14, 16-19]. Consequently, there is need to review the existing studies linking OCT1 variants and metformin responses in order to (a) summarize the overall effects of OCT1 polymorphisms on therapeutic responses elicited by metformin intake and (b) to evaluate the potential role of such polymorphisms in terms of interethnic differences in response to metformin therapy.

\section{Previous literatures}

Preliminary studies have reported the effects of OCT1 variants on metformin responses in T2DM individuals $[16,20-24]$. In a meta-analysis carried out by Dujic et al. (2017), in order to clarify the significance of genetic variations of metformin transporter genes on glycemic response to metformin, nine candidate variants in membrane transporter genes (thereof 3 variants from OCT1) were analyzed in 7968 individuals across the cohorts of the Metformin Genetics consortium (MetGen). The authors show that the candidate variants in membrane transporter genes showed no significant effect on metformin response assessed as HbA1c reduction in patients with T2DM [14].

\section{Why we will conduct this systematic review?}

The previous meta-analysis studied the effects of three candidate OCT1 variants on glycemic response to metformin in 7968 MetGen participants of European ancestry.

- Our study will not be restricted on pre-specified OCT1 variants. We will analyze every OCT1 variant identified that could be related to metformin response. Of note, Seitz et al. performed a global scale population analysis of OCT 1 variants and identified 85 variants in 52 worldwide population groups that included sub-Saharan Africa, the Middle East and North Africa, Central Asia, East Asia and Oceania, Europe, and America [15].

- Our study will add studies that were not included in the previous meta-analysis [17, 22-26].

- Genetic variation frequencies differ among different ethnicities, which may be associated with variation of susceptibility to adverse drug reactions among the different populations [27]. In this study, we will also compare the allele frequency distribution of OCT1 genetic variants among different ethnicities.

\section{Objective}

The objective of this systematic review is to highlight and summarize the overall effects of OCT1 polymorphisms on therapeutic responses to metformin and to evaluate their potential roles in terms of interethnic differences with metformin therapy.

\section{Methods}

This systematic review protocol is reported following the Preferred Reporting Items for the Systematic Reviews and Meta-analysis Protocols (PRISMA-P) 2015 Checklist (Additional file 1) [28]. 


\section{Type of participants}

Participants included in eligible studies must be diagnosed with T2DM and treated with metformin monotherapy for at least 3 months.

Most guidelines (American Diabetes Association, European Association for the Study of Diabetes, American Association of Clinical Endocrinologists) for the management of T2DM recommend an initial approach consisting of lifestyle changes and monotherapy, preferably with metformin. This recommendation is applicable if a patient is diagnosed with an initial HbA1c less than $9 \%$ and no existing contraindications (eGFR $<30 \mathrm{ml} / \mathrm{min} / 1.73 \mathrm{~m} 2$ ) [29]. Three-month period on metformin monotherapy is reasonable to assess for glycemic improvement. Treatment modification is recommended when the HbAlc target (HbA1c $>7 \%$ ) is not achieved or maintained by metformin monotherapy at maximal tolerated dose over 3 to 6 months [2].

\section{Type of exposure}

We will include studies in which participants were genotyped to investigate genetic variants of OCT1.

\section{Comparators}

The comparators are the responders and non-responders to metformin treatment. Response to metformin will be graded based on HbA1c level. Non-responders will constitute patients whose HbA1c levels declined by less than $1 \%$ after 3 months of treatment. Responders will be cases where HbA1c levels decreased by $1 \%$ or more after 3 months of treatment.

\section{Outcomes}

The primary outcome is the clinical effect of OCT1 polymorphisms on metformin response. Genetic polymorphisms of OCT1 will include single-nucleotide polymorphisms (SNPs), deletions, duplications, and copy-number variants. Where possible, effect estimate will include odds ratio and relative risk for the genetic variant effects in responders compared to non-responders.

Secondary outcomes include effects of OCT1 polymorphisms on fasting plasma glucose (FPG) and postprandial plasma glucose (PPG) after treatment with metformin monotherapy. FPG and PPG will be compared between responders and non-responders. The incidence of gastrointestinal side-effects will be also compared between responders and non-responders where possible.

\section{Eligibility criteria \\ Inclusion criteria}

- Cross-sectional, case-control, and cohort studies assessing the genetic effects of OCT1 variants on metformin responses $(\mathrm{HbA} 1 \mathrm{c}$, fasting plasma glucose levels, post-prandial glucose levels, and gastrointestinal side effects including nausea, vomiting, and diarrhea) in T2DM individuals, published between January 1990 and July 2017 without any geographical restriction

- Studies published in English or French

- Studies in which participants received metformin monotherapy as initial anti-hyperglycemic therapy for at least 3 months

\section{Exclusion criteria}

We will not consider:

- Letters, reviews, case reports, editorials, and comments

- Studies conducted with normal or pre-diabetes participants. Pre-diabetes is defined according to the following criteria: impaired fasting blood glucose (IFG) values between 100 and $125 \mathrm{mg} / \mathrm{dL}$ after at least $8 \mathrm{~h}$ of fasting, and/or glucose intolerance (ITG) when glycaemia values are between 140 and $199 \mathrm{mg} / \mathrm{dL} 2 \mathrm{~h}$ post oral administration of a $75 \mathrm{~g}$ glucose load (OGTT), and/or if the values of glycosylated hemoglobin (HbA1c) are between 5.7 and 6.4\%

- Studies in which participants received only one dose of metformin

- Studies in which participants also have other conditions like chronic gastrointestinal diseases, chronic liver disease, cholelithiasis, chronic pancreatitis, inflammatory bowel disease, gastroduodenal ulcer, chronic kidney disease, and endocrine disorders

- Studies in which relevant data on metformin responses is lacking or impossible to extract

\section{Search strategy for identifying relevant studies} We will search the following electronic databases: MEDLINE via PubMed, Embase, and CINAHL from January 1990 to July 2017 without any geographical restrictions. The choice of 1990 as onset date was made on the basis that OCT1 was cloned and functionally characterized in the early-mid-1990s [30, 31]. The search strategy based on the combination of relevant terms will be designed by a librarian. The main search strategy conducted in MEDLINE via PubMed is shown in Table 1. This search strategy will be adapted for possible extension to other databases and will be updated as we progress through the review. We will also manually search reference lists from relevant studies and contact experts in the field in order to identify additional eligible studies.

\section{Data collection and analysis}

\section{Selection of studies for inclusion in the review}

Two review authors (EPMM and MGF) will independently identify articles and sequentially screen their titles 
Table 1 Search strategy in MEDLINE/PubMed

\begin{tabular}{ll}
\hline Search & Search terms \\
\hline$\# 1$ & "type 2 diabetes" OR Diabetes (MeSH Terms) \\
$\# 2$ & "genetic markers" OR "genetic polymorphism" OR "Single \\
& nucleotide polymorphism" OR "Polymorphism" OR "variant" \\
& OR "gene" OR "allele" OR Genetic (MeSH Terms) \\
\#3 & "Organic Cation Transporter 1" OR "OCT 1" OR solute carrier \\
& family 22 organic cation transporter, member 1 (MeSH Terms) \\
\#4 & \#1 AND \#2 AND \#3 \\
\hline
\end{tabular}

and abstracts for eligibility. Thereafter, full texts of articles deemed potentially eligible will be retrieved. Further, these review authors will independently assess eligibility for inclusion in the review based on the inclusion and exclusion criteria. Any disagreements between the two review authors will be resolved by consensus and arbitration of a third review author if necessary. A PRISMA (Preferred Reporting Items for Systematic Review and Meta-Analysis) flow diagram [32] will document the process of literature selection and reasons for exclusion.

\section{Data extraction and management}

Two review authors (EPMM and MGF) will independently extract data in accordance with the methods outlined in the Cochrane Handbook for systematic reviews of interventions. A data extraction form will be designed. Approximatively $10 \%$ of the eligible studies will be randomly selected and used as pilot in the data extraction sheet in order to ensure its suitability.

Data will be collected on the first author name, year of publication, geographical location (country where the study was performed), study design, sample size, participant characteristics (mean or median age, age range, proportion of males), duration of treatment with metformin monotherapy, relevant OCT1 polymorphism, minor allelic frequencies in each population with Hardy Weinberg equilibrium if available, and primary outcome measurements (measure of metformin response after treatment with metformin). Gastrointestinal side effects, glycated hemoglobin A1 (HbA1c) levels, fasting plasma glucose (FPG), and post-prandial plasma glucose (PPG) concentrations after the treatment with metformin will be used as indices for metformin responses. Any disagreements between the two review authors will be resolved through discussion and by consulting a third author if necessary. Should any article be duplicated, we will contact the corresponding author and include the more relevant version. For managing missing data, we will contact the corresponding author of the respective studies in an attempt to obtain the required details. If no correspondence is received, the study will be included in the systematic review and discussed in the narrative summary.
Data analysis including assessment of heterogeneity

Study characteristics and the effect estimates of OCT1 polymorphisms on metformin responses will be presented in full, in tabular form. Since this effect varies from one study to another, we will derive the pooled estimate of each polymorphism investigated in multiple studies $(\geq 2)$, by using a random effect model.

Effects of potential confounding variables associated with metformin responses including metformin dosing, duration of treatment with metformin monotherapy, lifestyle changes, and drugs interactions will be dealt by using multivariable meta-regression analysis. All statistical analyses will be carried out using Stata statistical software version 14 (Stata Corporation, College Station, Texas, USA).

Statistical heterogeneity among the included studies will be assessed by the $X^{2}$ test on Cochrane's Q statistic. A $P$ value less than 0.1 will indicate significant heterogeneity. The $I^{2}$ statistic test will be further used to quantify the heterogeneity in the measure of association across studies. Values of $25 \%, 50 \%$, and $75 \%$ for $I^{2}$ will represent, respectively, low, medium, and high heterogeneity [33]. Where substantial heterogeneity is detected, a subgroup analysis will be performed to investigate the possible sources of heterogeneity using the following grouping variables: metformin dosing, sample size, lifestyle changes, practice of physical activity, and genotyping methods. If included studies differ significantly in design, sampling, and outcome measures, a narrative synthesis of the findings will be provided.

\section{Subgroup analysis}

We will conduct a subgroup analysis based on metformin dosing, sample size, lifestyle changes, and the genotyping method used to detect genetic variants. For the factor metformin dosing, $<1.500 \mathrm{mg}$ vs $>1.500 \mathrm{mg}$ will be compared. For the sample size, small vs large will be compared. For lifestyle changes, be on diet (yes vs no) and practice of physical activity (yes vs no) will be compared. For the genotyping methods, used Taqman vs others methods will be compared. Pooled odds ratios (ORs) and 95\% confidence intervals (CI) in each subgroup will be calculated. The heterogeneity between subgroups will be detected by using the $X^{2}$ test on Cochrane's Q statistic.

\section{Assessment of publication and reporting biases}

We will assess publication bias by using standard approaches including Funnel Plots and Egger tests if enough eligible studies are available [34]. The reporting quality of each study will be independently assessed by two review authors (EPMM and MGF) using the STREGA (Strengthening the Reporting of Genetic Association 
Studies) statement [35] which offers guidelines for reporting of individual genetic association studies.

\section{Assessment of methodological and evidence qualities}

Studies deemed fit for inclusion in the systematic review will be scored for methodological quality using the Newcastle-Ottawa assessment scale (NOS) [36]. Each domain will be rated with "high," "low," or "unclear" with regard to the risk of bias, with free text explanations.

We will also use the Grading of Recommendations Assessment, Development, and Evaluation (GRADE) methodology to assess the quality of evidence for each outcome. Quality rating of overall evidence will be downgraded according to five factors: risk of bias, inconsistency, indirectness, imprecision, and publication bias. In addition and where appropriate, the reasons to upgrade the evidence quality will include a large magnitude of effects, a dose-response gradient, and plausible residual confounding that would reduce a demonstrated effect or suggest a spurious effect when results show no effect. We will integrate downgrading and upgrading factors to obtain an overall quality of evidence for each outcome of interest. Overall quality of evidence will be then ranked as high, moderate, low, or very low as specified by the GRADE approach $[37,38]$.

\section{Discussion}

Type 2 diabetes a chronic degenerative metabolic disease represents a major medical and public health problem. After lifestyle changes failure, metformin is prescribed as first-line treatment for T2DM. However, metformin is not a panacea. Clinical practice indicates that there are considerable inter-individual variations in metformin response, with about $35 \%$ of patients failing to achieve initial glycemic control on metformin monotherapy $[39,40]$. Several lines of evidence based on pharmacogenetic research have demonstrated that genetic variation is one of the major factors affecting metformin responses [41]. In addition, it is well known that genetic polymorphisms in gene encoding drug-metabolism enzymes and drug transporters contribute to interindividual variabilities in the pharmacokinetics/pharmacodynamics profiles of clinical drugs [42]. The most studied transporter regarding the impact of genetic variation on metformin action has been OCT1. The gene encoding OCT1 is highly polymorphic, with a number of coding missense single nucleotide polymorphisms that affect its activity and function [43]. This study will systematically review worldwide reports that have investigated an association between any OCT1 genetic polymorphism and therapeutic response to metformin in T2DM patients. Understanding the diversity of genetic markers associated with drug response across different global populations is essential to set ethnicity-specific reference for adverse drug reactions and identify patient groups whose genetic characteristics put them at special risk from either excessive or reduced pharmacologic effects of metformin. In terms of limitations, definitive conclusions may not be possible due to the small sample size and heterogeneity of study design and protocols.

\section{Additional file}

Additional file 1: PRISMA-P 2015 checklist. (DOC 82 kb)

\section{Abbreviations}

AMPK: Adenosine monophosphate-activated protein kinase; FPG: Fasting plasma glucose; HbA1c: Glycosylated hemoglobin; LKB1: Liver kinase B1; OCT1: Organic cation transporter 1; OCTs: Organic cation transporters; PPG: Postprandial plasma glucose; PRISMA: Preferred Reporting Items for Systematic Review and Meta-Analysis; STREGA: Strengthening the Reporting of Genetic Association Studies; TDM: Type 2 diabetes mellitus

\section{Acknowledgements}

We are grateful to the Organization of Women in Science for the Developing World (OWSD) and Swedish International Development Cooperation Agency (SIDA) for their support.

Availability of data and materials

All available data can be obtained by contacting the corresponding author.

Authors' contributions

EPMM conceived and designed the protocol and drafted and revised the manuscript for intellectual content. MGF designed the protocol and revised the manuscript for intellectual content. MFE supervised and revised the manuscript for intellectual content. PMOO supervised and revised the manuscript for intellectual content. All authors had full access to the data. EPMM is the guarantor of the review. All authors approved the final version of the submitted manuscript.

Ethics approval and consent to participate

Ethical approval is not required for this study as it is a systematic review.

Consent for publication

Not applicable.

Competing interests

The authors declare that they have no competing interests.

\section{Publisher's Note}

Springer Nature remains neutral with regard to jurisdictional claims in published maps and institutional affiliations.

\section{Author details \\ ${ }^{1}$ Molecular and Clinical Pharmacology Research Laboratory, Department of Pharmacology, Discipline of Pharmaceutical Sciences, School of Health Sciences, University of KwaZulu-Natal, PO Box X5401, Durban, South Africa. ${ }^{2}$ Laboratory of Public Health Research Biotechnology (LAPHER-Biotech), Biotechnology Centre, University of Yaounde I, PO Box 3851, Yaounde, Cameroon. ${ }^{3}$ Laboratory of Molecular Medicine and Metabolism (LMMM), Biotechnology Centre, University of Yaounde I, PO Box 3851, Yaounde, Cameroon. ${ }^{4}$ Cardio-Metabolic Research Group (CMRG), Department of Physiological Sciences, Stellenbosch University, Stellenbosch, South Africa.}

Received: 21 February 2018 Accepted: 10 July 2018

Published online: 25 July 2018

\section{References}

1. American Diabetes Association. Pharmacologic approaches to glycemic treatment. Diabetes Care. 2017;40(Suppl 1):64-74. 
2. Inzucchi SE, Bergenstal RM, Buse JB, Diamant M, Ferrannini E, Nauck M, et al. Management of hyperglycemia in type 2 diabetes: a patient-centered approach: position statement of the American Diabetes Association (ADA) and the European Association for the Study of Diabetes (EASD). Diabetes Care. 2012;35:1364-79.

3. Foretz M, Hebrard S, Leclerc J, Zarrinpashneh E, Soty M, Mithieux G, et al. Metformin inhibits hepatic gluconeogenesis in mice independently of the LKB1/AMPK pathway via a decrease in hepatic energy state. J Clin Invest. 2010;120:2355-69.

4. Pawlyk AC, Giacomini KM, McKeon C, Shuldiner AR, Florez JC. Metformin pharmacogenomics: current status and future directions. Diabetes. 2014;63: 2590-9.

5. Madiraju AK, Erion DM, Rahimi Y, Zhang XM, Braddock DT, Albright RA, et al. Metformin suppresses gluconeogenesis by inhibiting mitochondrial glycerophosphate dehydrogenase. Nature. 2014;510:542-6.

6. Shu Y, Sheardown SA, Brown C, Owen RP, Zhang S, Castro RA, et al. Effect of genetic variation in the organic cation transporter 1 (OCT1) on metformin action. J Clin Invest. 2007;117:1422-31.

7. Higgins WJ, Bedwell DW, Zamek-Gliszczynski MJ. Ablation of both Oct1 and Oct2 alters metformin pharmacokinetics but has no effect on tissue drug exposure and pharmacodynamics. Drug Metab Dispos. 2012;40:1170-7.

8. Koepsell H, Lips K, Volk C. Polyspecific organic cation transporters: structure, function, physiological roles, and biopharmaceutical implications. Pharm Res. 2007;24:1227-51.

9. Nies AT, Schaeffeler E, van der Kuip H, Cascorbi I, Bruhn O, Kneba M, et al. Cellular uptake of imatinib into leukemic cells is independent of human organic cation transporter 1 (OCT1). Clin Cancer Res. 2014;20:985-94.

10. Meyer-Wentrup F, Karbach U, Gorboulev V, Arndt P, Koepsell H. Membrane localization of the electrogenic cation transporter rOCT1 in rat liver. Biochem Biophys Res Commun. 1998;248:673-8.

11. Koepsell H. The SLC22 family with transporters of organic cations, anions and zwitterions. Mol Asp Med. 2013;34:413-35.

12. Cook MN, Girman CJ, Stein PP, Alexander CM. Initial monotherapy with either metformin or sulphonylureas often fails to achieve or maintain current glycaemic goals in patients with type 2 diabetes in UK primary care. Diabet Med. 2007;24:350-8

13. Maruthur NM, Gribble MO, Bennett WL, Bolen S, Wilson LM, Balakrishnan P, et al. The pharmacogenetics of type 2 diabetes: a systematic review. Diabetes Care. 2014;37:876-86.

14. Dujic T, Zhou K, Yee SW, van Leeuwen N, de Keyser CE, Javorský M, et al. Variants in pharmacokinetic transporters and glycemic response to metformin: a metgen meta-analysis. Clin Pharmacol Ther. 2017;101:763-72.

15. Seitz T, Stalman R, Nawar D, Chen J, Pojar S, Dos Santos Pereira JN, et al. Global genetic analyses reveal strong inter-ethnic variability in the loss of activity of the organic cation transporter OCT1. Genome Med. 2015;7:1-23.

16. Zhou Y, Ye W, Wang Y, Jiang Z, Meng X, Xiao Q, et al. Genetic variants of OCT1 influence glycemic response to metformin in Han Chinese patients with type-2 diabetes mellitus in Shanghai. Int J Clin Exp Pathol. 2015;8: 9533-42.

17. Tarasova L, Kalnina I, Geldnere K, Bumbure A, Ritenberga R, Nikitina-Zake L, et al. Association of genetic variation in the organic cation transporters $\mathrm{OCT} 1, \mathrm{OCT} 2$ and multidrug and toxin extrusion 1 transporter protein genes with the gastrointestinal side effects and lower BMI in metformin-treated type 2 diabetes patients. Pharmacogenet Genomics. 2012;22:659-66.

18. Becker ML, Visser LE, van Schaik RH, Hofman A, Uitterlinden AG, Stricker BH, et al. Genetic variation in the organic cation transporter 1 is associated with metformin response in patients with diabetes mellitus. Pharmacogenomics J. 2009;9:242-27.

19. Dujic T, Zhou K, Donnelly LA, Tavendale R, Palmer CN, Pearson E, et al. Association of organic cation transporter 1 with intolerance to metformin in type 2 diabetes: a GoDARTS study. Diabetes. 2014;64:1786-93.

20. Chen L, Takizawa M, Chen E, Schlessinger A, Segenthelar J, Choi JH, et al. Genetic polymorphisms in organic cation transporter 1 (OCT1) in Chinese and Japanese populations exhibit altered function. J Pharmacol Exp Ther. 2010;335:42-50.

21. Becker ML, Visser LE, Van Schaik RH, Hofman A, Uitterlinden AG, Stricker BH. Interaction between polymorphisms in the OCT1 and MATE1 transporter and metformin response. Pharmacogenet Genomics. 2010;20:38-44.

22. Christensen MMH, Højlund $K$, Hother-Nielsen O, Stage TB, Damkier $P$, Beck-Nielsen $\mathrm{H}$, et al. Steady-state pharmacokinetics of metformin is independent of the OCT1 genotype in healthy volunteers. Eur J Clin Pharmacol. 2015;71:691-7.

23. Xiao D, Guo Y, Li X, Yin J-Y, Zheng W, Qiu X-W et al. The impacts of SLC22A1 rs594709 and SLC47A1 rs2289669 polymorphisms on metformin therapeutic efficacy in Chinese type 2 diabetes patients". Int J Endocrinol. 2016. (2016) 4350712. https://doi.org/10.1155/2016/4350712.

24. Sur D. A tale of genetic variation in the human Slc22a1 gene encoding OCT1 among type 2 diabetes mellitus population groups of West Bengal, India. IMPACT. 2014;2:97-106.

25. Christensen MMH, Brasch-Andersen C, Green H, Nielsen F, Damkier P, BeckNielsen $\mathrm{H}$, et al. The pharmacogenetics of metformin and its impact on plasma metformin steady-state levels and glycosylated hemoglobin A1c. Pharmacogenet Genomics. 2011;21:837-50.

26. Koshy M, Sethupathy S, Annamalai PT, Renju VC, Santha K. Association of oct1 gene polymorphism with glycemic status and serum metformin levels in type II diabetes mellitus patients. Int J Pharm Sci Res. 2013;4:1940-5.

27. Bachtiar M, Lee CGL. Genetics of population differences in drug response. Cur Genet Med Rep. 2013;1:162-70.

28. Shamseer L, Moher D, Clarke M, Ghersi D, Liberati A, Petticrew M, et al. Preferred reporting items for systematic review and meta-analysis protocols (PRISMA-P) 2015: elaboration and explanation. BMJ. 2015;349:97647.

29. Inzucchi SE, Bergenstal RM, Buse JB, Diamant M, Ferrannini E, Nauck M, et al. Management of hyperglycaemia in type 2 diabetes, 2015: a patient-centred approach. Update to a position statement of the American Diabetes Association and the European Association for the Study of Diabetes. Diabetologia. 2015;58:429-42.

30. Gründemann D, Gorboulev V, Gambaryan S, Veyhl M, Koepsell H. Drug excretion mediated by a new prototype of polyspecific transporter. Nature. 1994;372:549-52.

31. Okuda M, Saito H, Urakami Y, Takano M, Inui K. cDNA cloning and functional expression of a novel rat kidney organic cation transporter, OCT2. Biochem Biophys Res Commun. 1996;224:500-7.

32. Moher D, Liberati A, Tetzlaff J, Altman DG. PRISMA group preferred reporting items for systematic reviews and meta-analyses: the PRISMA statement. PLoS Med. 2009;6:e1000097.

33. Higgins JP, Thompson SG. Quantifying heterogeneity in a meta-analysis. Stat Med. 2002;21:1539-58.

34. Egger M, Davey Smith G, Schneider M, Minder C. Bias in meta-analysis detected by a simple, graphical test. BMJ. 1997;315:629-34

35. Little J, Higgins JP, loannidis JP, Moher D, Gagnon F, von Elm E, et al. STrengthening the REporting of Genetic Association Studies (STREGA)--an extension of the STROBE statement. PLoS Med. 2009;6:e1000022.

36. Wells $G$, Shea B, O'Connell D, Peterson J, Welch V, Losos M et al. The Newcastle-Ottawa Scale (NOS) for assessing the quality of nonrandomised studies in meta-analyses, 2013. http://www.ohri.ca/programs/clinical_ epidemiology/oxford.asp. Accessed 23 Oct 2017.

37. Higgins JPT, editors., Cochrane Handbook for Systematic Reviews of Interventions Version 5.1.0 [updated March 2011]. The Cochrane Collaboration; 2011. Available from: www.handbook.cochrane.org.

38. Guyatt G, Oxman AD, Akl EA, Kunz R, Vist G, Brozek J, et al. GRADE guidelines: 1. Introduction-GRADE evidence profiles and summary of findings tables. J Clin Epidemiol. 2011;64:383-94.

39. Kahn SE, Haffner SM, Heise MA, Herman WH, Holman RR, Jones NP, et al. Glycemic durability of rosiglitazone, metformin, or glyburide monotherapy. N Engl J Med. 2006:355:2427-43.

40. Chan SL, Samaranayake N, Ross CJD, Toh MT, Carleton B, Hayden MR, et al. Genetic diversity of variants involved in drug response and metabolism in Sri Lankan populations: implications for clinical implementation of pharmacogenomics. Pharmacogenet Genomics. 2016;26:28-39.

41. Chen S, Zhou J, Xi M, Jia Y, Wong Y, Zhao J, et al. Pharmacogenetic variation and metformin response. Cur Drug Metabol. 2013;14:1070-82.

42. Takane H, Shikata E, Otsubo K, Higuchi S, leiri I. Polymorphism in human organic cation transporters and metformin action. Pharmacogenomics. 2008:9:415-22.

43. Florez JC. The pharmacogenetics of metformin. Diabetologia. 2017;60:1648-55. 